
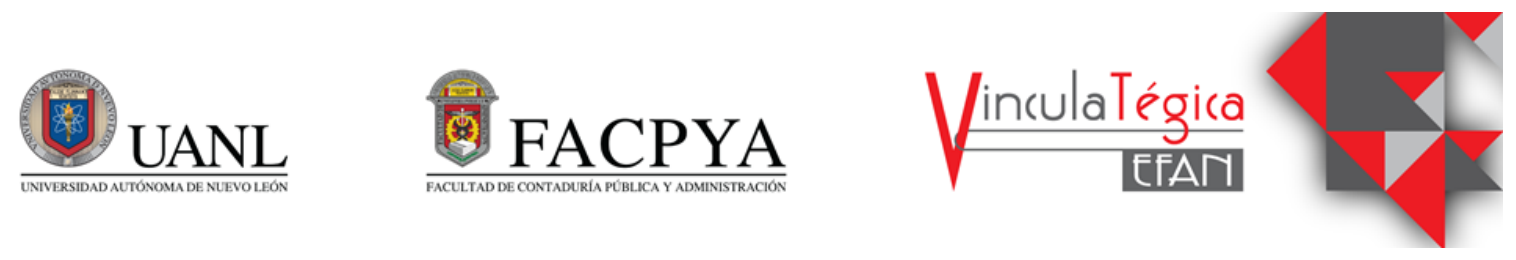

\title{
Impacto del ecommerce mediante plataformas digitales en México
}

\author{
Diana Maricela Vasquez Treviño ${ }^{1}$, Luis Vicente Cabeza Llanos $^{2}$ y Juan Patricio Galindo \\ Mora $^{3}$ \\ ${ }^{1}$ Universidad Autónoma de Nuevo León, FACPYA email: diana.vasqueztrv@uanl.edu.mx \\ ${ }^{2}$ Universidad Autónoma de Nuevo León, FACPYA email: luisvcabeza@gmail.com \\ ${ }^{3}$ 1Universidad Autónoma de Nuevo León, FACPYA email: juan.galindomr@uanl.edu.mx
}

Información del artículo revisado por pares

Fecha de aceptación: junio-2021

Fecha de publicación en línea: diciembre-2021

DOI: https://doi.org/10.29105/vtga7.2-60

\section{Resumen}

En este trabajo se hizo una revisión teórica sobre el impacto del ecommerce en la economía mexicana, donde se identificaron las ventajas, desventajas, así como su evolución en los últimos años. Las empresas están utilizando el ecommerce como estrategia para aumentar las ventas y salir de la crisis económica derivada del covid 19.

Palabras clave: Ecommerce, pymes, plataformas digitales.

\section{INTRODUCCIÓN}

El año 2020 fue marcado por la pandemia del COVID 19, Alvarado y Cabrera (2020) mencionan en su investigación sobre la recesión económica y la pérdida de empleos que la economía mexicana tras el cierre económico de actividades no esenciales por la Jornada Nacional de sana distancia tuvo un impacto negativo. Sectores como el manufacturero, servicios y turístico fueron severamente golpeados y generaron miles de pérdidas de empleos en México.

El gobierno mexicano se vio en la necesidad de implementar medias para contrarrestar la expansión del nuevo coronavirus; entre las acciones
In this article the impact of e-commerce on the mexican economy was analyzed. The advantages and disadvantages were identified, and its evolution on the last few years. Companies are using ecommerce as a strategy to increase their sales and get out of the economic crisis created after Covid-19 pandemic.

Keywords: Ecommerce, pymes, digital media JEL: L81, M10

implementadas destacan el distanciamiento social y el cierre temporal de comercios con punto físicos de venta de productos y servicios al público y a las empresas.

Con las restricciones previamente descritas, las empresas mexicanas se vieron forzadas a ofrecer sus productos mediante el comercio digital (ecommerce), tras el cierre temporal repentino de los comercios surgieron las siguientes preguntas: ¿las empresas mexicanas están preparadas para adoptar el ecommerce? ¿Desde la perspectiva teórica se puede analizar el impacto económico de ecommerce en México? 
En esta investigación hablaremos sobre el ecommerce. Para analizar este tema es necesario mencionar sus características, ventajas y desventajas, mostrar las estrategias y el crecimiento que conllevan para la adaptación en las empresas. Además, se analizarán a los principales autores y la evolución que han tenido en México.

El objetivo de esta investigación es identificar las variables que impactan para la adaptación del ecommerce en empresas y su impacto en la economía mexicana, así como mostrar el crecimiento que ha tenido en México. Esta investigación teórica va dirigida a personas y empresas que utilizan plataformas digitales en organizaciones para generar una ventaja competitiva e incrementar sus ventas para posicionar a su compañía en un mejor nivel.

\section{ANTECEDENTES TEÓRICOS}

Picazo Vela (2013) menciona en su estudio comercio electrónico y emprendimiento mencionan que las empresas ven al ecommerce como una oportunidad de abrir sus canales de distribución y aumentar sus ventas.

Desde la perspectiva empresarial, el ecommerce aumenta la competitividad entre las empresas minoritarias, amplia la elección entre los clientes y acota la gestión de la cadena de suministros de los productos (OECD, 2018). Según la estadística de la asociación mexicana de venta online (AMVO) 7 de cada 10 compradores en línea se sienten seguros al comprar en una plataforma digital y se ve reflejando en su experiencia positiva y su motivación en seguir comprando. (AMVO, 2020).

Turban y Leen (2000) definen el ecommerce bajo los siguientes enfoques:

- Desde un enfoque de comunicaciones: "eCommerce es la entrega de información, productos/servicios, o pagos sobre una línea telefónica, red de computadoras o cualquier otro medio electrónico."

- Desde un enfoque de proceso de negocios: "e-Commerce es la aplicación de tecnologías que apuntan a la automatización de transacciones de negocios y su flujo de trabajo".

- Desde el enfoque de servicios: "eCommerce es una herramienta que aborda el deseo de las firmas, consumidores y administración de reducir costos, mejorando la calidad de bienes, incrementando la velocidad del servicio de entrega".

- Desde el enfoque de informática: "eCommerce adquiere la capacidad de comprar y vender productos e información en la Internet y otros servicios en línea.

Para esta investigación utilizaremos la siguiente definición sobre el ecommerce: toda transacción de negocios que se realice por medio de plataformas digitales que ayude al fortalecimiento de las relaciones comerciales y aumente las oportunidades de negocio para fomentar la economía (Cuellar y Rivas, 2005)

En el informe del INEGI (2018) describen que el valor agregado bruto del comercio electrónico es el primer indicador de la economía digital específicamente el ecommerce, entendido transacción de compra venta a través de plataformas digitales, cuyo pago puede ser hecho en línea, o no, y ayuda en la participación del Producto Interno Bruto (PIB) reportando un crecimiento del 2 al 3\% en el comercio electrónico en los últimos 5 años.

El desarrollo acelerado de la tecnología digital tiene un efecto positivo sobre la economía además abre las puertas a nuevos negocios para la penetración de nuevos mercados, además forma el escenario idóneo para hacer nuevas relaciones comerciales fortaleciendo la comunicación y superando la barrera de la distancia. (Coyle, 2016).

\section{Problemas de Investigación}

A pesar de que en México el $61.1 \%$ de los mexicanos posee una conexión a Internet (un porcentaje mayor al promedio mundial) aún hay zonas del territorio con una pobre calidad de conexión a internet que limita a 
los consumidores. Para poder poner a México al nivel de los países con economías desarrolladas es crucial cerrar esta brecha de desigualdad digital, no tener el acceso a una mejor conectividad pone a las empresas en una situación de desigualdad en la que se pierden muchas oportunidades de desarrollo económico. (AMVO, 2019).

Otro factor que afecta al ecommerce es la confianza en el proceso de entrega en una compra en línea, esta es un área de oportunidad para que quienes no son compradores en línea entiendan y disfruten los beneficios del ecommerce. Para el 79\% de estas personas es el limitante principal el temor de que no llegue su compra. (AMVO, 2019).

Palacios (2006) tiene como principal limitación de las PYMES en la implantación del ecommerce en México los recursos económicos para invertir en infraestructura tecnológica y una visión carente de su alcance.

\section{Hipótesis}

El ecommerce mediante plataformas digitales está impactando positivamente la economía mexicana

\section{MARCO TEÓRICO} Plataformas digitales en México

En 1990 surgió la revolución digital con la llegada de las computadoras y con la democratización del acceso a internet. La innovación tecnológica adquirió el adjetivo "digital" y marco una diferencia entre el mundo tradicional y el mundo digital donde las empresas y las naciones tendrían que adaptarse a la nueva era digital de lo contrario, no podrían competir y quedarían fuera del mercado" (Mochón, 2014)

La encuesta nacional sobre disponibilidad y uso de tecnologías de la Información en los hogares (ENDUTIH) menciono en su encuesta que el $65 \%$ de la población mayor a 6 años son usuarios de Internet y por lo tanto también a plataformas digitales. (INEGI, 2018).

El estudio de la Inversión en comunicación en Internet en México, la publicidad en plataformas digitales aumento un 21.8\% (análisis del PwC) en el año 2016 donde se muestran que las empresas invierten más recursos en la publicidad en línea, principalmente en redes sociales y motores de búsqueda. Otro indicador para el acceso a internet es el uso de la banda ancha móvil, el cual presento un aumento. (OECD, 2018).

Cabe señalar que México según el estudio de mercado de IAB México (Interactive Advertising Bureau) tiene el $60 \%$ de penetración de usuarios en Internet en el país a diferencia de Estados Unidos de América con un $80 \%$ y el $44 \%$ de los mexicanos cuentan un smartphone (teléfono inteligente) en el 2016. Dichas estadísticas son muy alentadoras para la implementación de comercio digital.

Molla y Licket (2005) mencionan las siguientes variables: infraestructura económica, tecnológica, jurídica y financiera como principales determinantes de la adopción del comercio electrónico en países en vías de desarrollo, siendo los recursos tecnológicos los de mayor impacto para la implementación del ecommerce.

En la Tabla 1 se muestra la clasificación de Turban y Lee (2000), donde se agrupa al comercio digital de acuerdo con la transacción y su definición.

Otro de los factores que impacta el ecommerce es el transporte, Andrés MuñozVillamizar et al 2020, en su estudio sobre el impacto del envió rápido en el ecommerce menciona que se ha elevado el costo del flete al consumidor final derivado al aumento de frecuencia de los transportes por entregar el producto el mismo día y al no utilizar las capacidades completas de las cargas de los camiones aunado a las ineficientes rutas de distribución. 


\section{Crecimiento de las plataformas digitales} en México

Medina Cadena menciona en un artículo publicado en el 2016 por la revista Forbes, la empresa global de consultoría y tecnología "Indra" que México está teniendo un crecimiento exponencial del comercio digital en los 5 últimos años, ya que reporto un crecimiento del 200\% del 2011 al 2016.

El avance tecnológico seguirá evolucionando y permitirá la relación entre más mercados teniendo mayor conciencia sobre la información del cliente. La constante es y seguirá siendo el cliente, los negocios deben centrarse cada vez más en las necesidades de los clientes el que, como y cuando el producto esté disponible en el mercado. (Deloitte, 2020).

La digitalización de la economía global es inevitable. Por tal motivo es necesario que las pymes mexicanas tomen como ejemplo la implementación de las nuevas plataformas digitales de los países desarrollas para evitar la laguna de capacitación y la aceptación de dicha tecnología por los usuarios. Además, al entrar a la nueva era tecnológica se abre el campo de competencia entre las compañías a nivel global (COFECE, 2020).

Con el desarrollo tecnológico acelerado de aplicaciones como páginas web, redes sociales, marketing digital y el cambio constante del gusto y las necesidades de los clientes las empresas deben de reaccionar rápido para ajustarse a la nueva tendencia del mercado y monitoreando constantemente los nuevos canales de comunicación (EUDE, 2019).

Los principales dispositivos a través de los cuales el consumidor accede a las compras online son los ordenadores, celulares inteligentes y tabletas digitales, y su frecuencia para realizar una compra en línea es en el orden que se mencionó, (AMVO, 2018) otro dato importante que revelo es que solo el $30 \%$ de las compras digitales en México pagan envió el consumidor.

\section{ecommerce}

Ventajas y desventajas del

La Comisión Nacional para la Protección y Defensa de los Usuarios de Servicios Financieros (CONDUSEF) detallan en su artículo "Lo que debes saber del comercio electrónico" alguna de las ventajas del ecommerce como:

- Servicio las horas del día los 365 días de la semana

- No hacer filas para pagar la cuenta

- Una comparación global del producto a comprar

- Disminución de tiempo para comprar el producto en el mercado

- aumenta las relaciones comerciales

- Aumenta la competitividad.

Ríos Ruiz 2015 menciona en su investigación que el ecommerce es una forma fácil, rápida, y eficiente de realizar compras y las principales ventajas que propone en su artículo son las siguientes:

- El cliente tiene acceso a la información disponible del producto desde un mismo lugar físico

- Es fácil comparar los productos de un mismo tipo en el mercado

- Elimina costos adicionales de búsqueda del mejor producto

- Disminuye el tiempo de transacciones comerciales

- Evita lugares conglomerados e inseguros

- Abre las puertas a nuevos mercados

- La publicidad tiene un menor costo

Algunas desventajas del ecommerce se mencionan a continuación: las compras en plataformas digitales tienen mayor riesgo ya que no existe un contacto personal, algunos comercios no cuentan con seguridad adecuada para procesar pagos electrónicos, algunas páginas no están en el idioma nativo del consumidos y puede causar confusión, aplicación del impuesto al valor agregado y 
uso de software y equipo de cómputo puede representar un alto costo para las empresas.

Una de las limitaciones del ecommerce en el mercado mexicano es el choque cultural de los emprendedores para transformar los negocios tradicionales en negocios digitales. Impulsar el desarrollo de tecnológico e innovación para fomentar el nuevo modelo de ventas a través de plataformas digitales. (Perdigón Llanes, 2018).

Otro de los factores determinantes para impulsar el ecommerce es la seguridad, se sebe crear una atmosfera de seguridad para que los consumidores y vendedores realicen las compras a través de medios digitales; la educación juega un papel determinante para el dominio de las plataformas tecnológicas y dar la seguridad para que la transacción del ecommerce sea un éxito.

\section{Las plataformas digitales estrategia comercial para superar la crisis económica derivada del COVID 19}

Rho Caterina; Fernández Raúl; Palma Brenda (2021) mencionan en su artículo un indicador de riesgo basado en el sentimiento para el sector financiero mexicano como la economía mexicana se están basando en datos no estructurados como las redes sociales y las plataformas digitales tienen injerencia en los bancos centrales y la supervisión financiera pueden verse afectados o beneficiados con el sentimiento del consumidor o de los inversionistas.

A pesar de la crisis económica es a nivel mundial, las compañías tecnológicas como Facebook, Apple, Microsoft, Amazon y Google han tenido un repunte en sus acciones debido a que las empresas las utilizan como estrategias de ventas por su bajo costo de implementación y su aceptación en el mercado. (CIO México, 2020).
CIO México (2020) menciona que Facebook presento un $18 \%$ de incremento en usuarios derivado a la sana distancia algunas de las actividades más usadas son las videollamadas, y los mensajes de texto que las pequeñas compañías utilizaron como medio de comunicación y estrategia para no verse afectadas por las medidas de sana distancia y el cierre temporal de espacio físicos en México.

Muñoz (2019) enfatiza como las empresas están generando un valor agregado mediante las plataformas digitales y la diferenciación que perciben los consumidores.

Otra de las plataformas digitales es el sistema bancario en México según Octavio Avendaño Carbellido que los menciona en su artículo los retos de la banca digital en México, se encuentra integrado por el Banco de México (Banxico), las instituciones de banca múltiple (IBM), las instituciones de banca de desarrollo y los fideicomisos públicos constituidos por el Gobierno federal, así como los organismos autorregulatorios bancarios (Asociación Mexicana de Bancos).

La evolución a las plataformas digitales ha dado paso a la apertura del ecommerce, uno de los pioneros en el desarrollo de estas plataformas son los bancos que han ayudado a los consumidores a agilizan los procesos de los usuarios y suman una parte vital en la sociedad. Entre las innovaciones se puede destacar las tarjetas digitales y las transacciones electrónicas.

\section{CONCLUSIONES}

Podemos concluir que México está afrontando una recesión económica derivado de la pandemia del COVID 19, y una de las estrategias de las empresas para contrarrestar esta situación es el ecommerce que ha impactado positivamente a la economía mexicana. Los nuevos canales digitales se han convertido en uno de los grandes y 
principales vías de ingresos de las empresas y estos son cada vez más confiables para los clientes.

Después de haber analizado el impacto del ecommerce en México, podemos aceptar teóricamente la hipótesis de esta investigación "El ecommerce mediante plataformas digitales está impactando positivamente la economía mexicana".

El desarrollo tecnológico seguirá su curso y cambiará la forma de hacer negocio entre las compañías. Los cambios serán más rápidos y tendrán menos duración, por lo que es importante que las compañías mexicanas estén a la vanguardia y busquen cumplir las necesidades de los clientes.

En las siguientes investigaciones se hará un instrumento para confirmar que las empresas mexicanas están utilizando las plataformas digitales como estrategia y ventaja competitiva para aumentar sus ventas y tener mayor penetración en el mercado.

Tabla 1 (Clasificación de e-Commerce por transacción).

\begin{tabular}{ll}
\hline \multicolumn{1}{c}{ TRANSACCIÓN } & \multicolumn{1}{c}{ Definición } \\
\hline $\begin{array}{l}\text { Negocio a Negocio } \\
\text { (Business-to-Business), }\end{array}$ & $\begin{array}{l}\text { Son las transacciones digitales entre las empresas, es de empresa a } \\
\text { empresa. }\end{array}$ \\
$\begin{array}{l}\text { Negocio-a-Consumidor } \\
\text { (Business-to- Consumer), }\end{array}$ & $\begin{array}{l}\text { Es la transacción directa entre empresa a cliente. Una de las plataformas } \\
\text { más usadas es Amazon y Mercado Libre. }\end{array}$ \\
$\begin{array}{l}\text { B2C } \\
\text { Consumidor-a-Consumidor } \\
\text { (Consumer-to-Consumer), }\end{array}$ & $\begin{array}{l}\text { Es la transacción de cliente a cliente, es la más utilizada, por ejemplo } \\
\text { C2C }\end{array}$ \\
$\begin{array}{l}\text { Fonsumidor-a-Negocio o los motores de búsqueda. } \\
\text { (Consumer-to-Business), }\end{array}$ & $\begin{array}{l}\text { Es la transacción que se hace de personas a negocios, las personas que } \\
\text { v2B }\end{array}$ \\
\hline Fuente: elaboración propia a partir de Turban y Lee (2000).
\end{tabular}

\title{
REFERENCIAS
}

\begin{abstract}
Alvarado, J. C. A., \& Tapia, C. F. J. C. LA RECESIÓN ECONÓMICA Y LA PÉRDIDA DE EMPLEOS EN MÉXICO A CAUSA DE LAS MEDIDAS DE SANA DISTANCIA IMPLEMENTADAS PARA MITIGAR LOS CONTAGIOS DEL NUEVO CORONAVIRUS COVID-19.
\end{abstract}

Avendaño Carbellido, O. (2018). Los retos de la banca digital en México. Revista IUS, 12(41), 87-108.

CIO México (05/06/2020) Las plataformas digitales, clave para superar el COVID-19 con éxito. http://cio.com.mx/las-plataformas-digitales-clave-para-superar-el-covid-19-con-exito/

COFECE. (2020). Estrategia Digital COFEC COFECE. (2020). Estrategia Digital COFECE. Comisión Federal de Competencia Económica, https://www.cofece.mx/wpcontent/uploads/2020/03/EstrategiaDigital_V10.pdf

Comisión Nacional para la Protección y Defensa de los Usuarios de Servicios 
Financieros. (CONDUSEF). Lo que debes de saber. https://www.condusef.gob.mx/?p=lo-quedebes-saber

Deloitte Touche Tohmatsu Limited. (2019). Comercio Electrónico. Antecedentes del uso de internet y comercio electrónico, https://www2.deloitte.com/content/dam/Deloitte/mx/Documents/consumerbusiness/2019/Comer cio-Electronico.pdf

Espinal, A. E., Gámez, J. I. G., \& Rodríguez, R. R. (2020). Fase actual del ciclo económico clásico en México y sus regiones: la recesión 2019-2020 en curso.

Geografía, E. D. N. I. Y. (s. f.). Comercio Electrónico. INEGI 2019.

https://www.inegi.org.mx/temas/vabcoel/

Gibbs, J., Kraemer, K. L., \& Dedrick, J. (2003). Environment and policy factors shaping global ecommerce diffusion: A cross-country comparison. The information society, 19(1), 5-18.

Koontz, H., Weihrich, H., \& Cannice, M. (2012). Administración una perspectiva global y empresarial. Perú: El comercio, S.A

Ley $N^{\circ} 1$. Ley Del Impuesto al Valor Agregado. México. (09/12/2019). http://www.diputados.gob.mx/LeyesBiblio/pdf/77_091219.pdf

López-Piñón, D. C., \& Terán-Cazares, M. M. (2020). Análisis de los factores que influyen en la intención de compra online.

Medina Cadena, S. (2018). Mochón, F. (2014). Economía Digital Curso MOOC. México: Alfaomega.

MÉXICO, I. (2010). Comercio electrónico como estrategia impulsora de las exportaciones en México. Aprendizaje Organizacional en las Mipymes, 3(3), 81.

Molla, A., \& Licker, P. S. (2005). eCommerce adoption in developing countries: a model and instrument. Information \& management, 42(6), 877-899.

Muñoz Cubides, N. K. M. (2019). Percepción del consumidor mexicano frente a la tendencia digital: E-commerce, por parte de la empresa de Retail: Wal-Mart.

OECD (2018) Plataformas digitales y competencia en México. http://oe.cd/dpcm

Palacios, J. J. (2003). The development of e-commerce in Mexico: A business-led passing boom or a step toward the emergence of a digital economy?. The information society, 19(1), 69-79.

Perdigón Llanes, R., Viltres Sala, H., \& Madrigal Leiva, I. R. (2018). Estrategias de comercio electrónico y marketing digital para pequeñas y medianas empresas. Revista Cubana de Ciencias Informáticas, 12(3), 192-208.

Picazo Vela, S., Ramírez Goni, P. N., \& Luna Reyes, L. F. (2013). Comercio electrónico y emprendimiento: un análisis aplicando la teoría del comportamiento planeado. RECAI. Revista de estudios en contaduría, administración e informática, (5), 1-20.

Muñoz-Villamizar, A., Velázquez-Martínez, J. C., Haro, P., Ferrer, A., \& Mariño, R. (2021). The 
environmental impact of fast shipping ecommerce in inbound logistics operations: A case study in Mexico. Journal of Cleaner Production, 283, 125400.

Ríos Ruíz, A. de los Ángeles. (2015). ANÁLISIS Y PERSPECTIVAS DEL COMERCIO ELECTRÓNICO EN MÉXICO. Perfiles De Las Ciencias Sociales, 3(5). https://revistas.ujat.mx/index.php/perfiles/article/view/993

Rho Caterina; Fernández Raúl; Palma Brenda (2021) Indicador de riesgo basado en el sentimiento para el sector financiero mexicano, Banco de México.

Tan, J., Tyler, K., \& Manica, A. (2007). Business-to-business adoption of eCommerce in China. Information \& management, 44(3), 332-351.

Zenvian 18 Mayo 2021, Plataformas multicanal y el e commerce: la fórmula perfecta para generar mayores ventas, La asociación mexicana de ventas online (AMVO) https://www.amvo.org.mx 